\title{
Potential authentication of various meat-based products using simple and efficient DNA extraction method
}

\begin{abstract}
Background: The growth of halal food consumption worldwide has resulted in an increase in the request for halal authentication. DNA-based detection using powerful real-time polymerase chain reaction (PCR) technique has been shown to be highly specific and sensitive authentication tool. The efficient DNA extraction method in terms of quality and quantity is a backbone step to obtain successful real-time PCR assays. In this study, different DNA extraction methods using three lysis buffers were evaluated and developed to recommend a much more efficient method as well as achieve a successful detection using real-time PCR. Results: The lysis buffer 2 (LB2) has been shown to be the best lysis buffer for DNA extraction from both raw and processed meat samples comparing to other lysis buffers tested. Hence, the LB2 has been found to be ideal to detect meat and porcine DNAs by real-time PCR using pairs of porcine specific primers and universal primers which amplified at $119 \mathrm{bp}$ fragment and $93 \mathrm{bp}$ fragment, respectively. This assay allows detection as low as $0.0001 \mathrm{ng}$ of DNA. Higher efficiency and sensitivity of real-time PCR via a simplified DNA extraction method using LB2 have been observed, as well as a reproducible and high correlation coefficient $(\mathrm{R} 2=0.9979)$ based on the regression analysis of the standard curve have been obtained. Conclusion: This study has established a fast, simple, inexpensive and efficient DNA extraction method that is feasible for raw and processed meat products. This extraction technique allows an accurate DNA detection by real-time PCR and can also be implemented to assist the halal authentication of various meat-based products available in the market. (C) 2019 Society of Chemical Industry.
\end{abstract}

